\title{
REPRESENTACIONES SOCIOCULTURALES DE GÉNERO EN ESTUDIANTES SECUNDARIOS/AS Y VIOLENCIAS DE GÉNERO EN LA ESCUELA
}

\author{
RHONNY LATORRE CHÁVEZ* \\ PABLo RoBledo VALLEJOS** \\ NiCOLÁS NiETO ARAOS***
}

\begin{abstract}
RESUMEN
El siguiente artículo se enmarca dentro de la investigación «Violencias de género: otra mirada a la brecha escolar» (2018), llevada a cabo por Fundación Semilla, y tiene por objetivo ahondar en la noción de representaciones socioculturales de género entendidas como los idearios, valoraciones e imaginarios que circundan a los roles de género- al interior de jóvenes estudiantes secundarios de la Región Metropolitana de Chile. Se ofrece una contextualización respecto al enfoque de género y su relación con las violencias en espacios escolares; se entregan referencias teóricas que sustentan el concepto propuesto; se analiza el comportamiento de las representaciones socioculturales al interior de la muestra y se propone un índice de representaciones como herramienta de análisis. El artículo concluye que la mayor parte de los/as jóvenes secundarios poseen representaciones inclusivas que cuestionan los roles estereotipados de género.
\end{abstract}

PALABRAS CLAVE: VIOLENCIAS DE GÉNERO, REPRESENTACIONES SOCIOCULTURALES DE GÉNERO, VIOLENCIA ESCOLAR

\footnotetext{
*Sociólogo de la Pontificia Universidad Católica de Chile. Investigador en Género de Fundación Semilla y del Centro de Reflexión e Investigaciones CRIN. Educador del Colegio Paulo Freire. Correo electrónico: r.latorre@fundacionsemilla.cl

**Sociólogo de la Universidad Alberto Hurtado, Chile. Magíster (c) en Antropologías Latinoamericanas. Investigador en Educación de Fundación Semilla e investigador en Pedagogías y Acción Colectiva en Kutral. Correo electrónico: p.robledo@fundacionsemilla.cl

***Sociólogo de la Universidad de Chile. Postítulo en Investigación y Acción en Mundos Juveniles. Investigador en Juventudes de Fundación Semilla. Docente de la carrera de Trabajo Social en la Universidad Alberto Hurtado, Chile. Correo electrónico: nieto@fundacionsemilla.cl
} 


\title{
REPRESENTAÇÕES SOCIOCULTURAIS DE GÊNERO EM ESTUDANTES DO ENSINO MÉDIO E VIOLÊNCIAS DE GÊNERO NA ESCOLA
}

\section{RESUMO}

O artigo a seguir faz parte da pesquisa "Violencias de Género: Otra mirada a la brecha escolar" (2018), realizada pela Fundação Semilla, e tem como objetivo aprofundar na noção de Representações Socioculturais de Gênero, - entendidas como os ideários, valorações e imaginários que envolvem os papéis de gênero - dentro dos jovens estudantes do ensino médio da região metropolitana do Chile. É oferecida uma contextualização sobre a abordagem de gênero e sua relação com as violências nos espaços escolares; são também entregues referências teóricas que sustentam o conceito proposto; é analisado o comportamento das representações socioculturais na amostra e é proposto um índice de representações como uma ferramenta de análise. $\mathrm{O}$ artigo conclui que a maioria dos e das jovens secundários têm representações inclusivas que questionam os papéis estereotipados de gênero.

\author{
PALAVRAS-CHAVE: VIOLÊNCIAS DE GÊNERO, REPRESENTAÇÕES SOCIOCULTURAIS DE \\ GÊNERO, VIOLÊNCIA ESCOLAR.
}

\section{SOCIO - CULTURAL REPRESENTATIONS OF GENDER IN SECONDARY SCHOOL STUDENTS AND GENDER VIOLENCE IN SCHOOL}

\begin{abstract}
The following article is a part of the research "Violencias de Género: Otra mirada a la brecha escolar" (2018) carried out by Semilla Foundation, and aims to delve into the notion of Socio-Cultural Representations of Gender, -defined as the ideals, valuations and imaginaries surrounding gender roles- within young secondary school students in the metropolitan region of Chile. The article offers a contextualization regarding the gender approach and its relation with school violence, offering theoretical references supporting the proposed concept, as well as an analysis of how socio-cultural representations behave in the sample, and an index of representations proposed as an analysis tool. The article concludes that the majority of the secondary school youth individuals are attached to inclusive representations of gender questioning stereotyped gender roles.
\end{abstract}

KEYWORDS: GENDER VIOLENCE, SOCIO-CULTURAL REPRESENTATIONS OF GENDER, SCHOOL VIOLENCE. 


\section{INTRODUCCIÓN}

Las representaciones sociales de género - entendidas como los idearios, valoraciones e imaginarios que circundan a los roles de género- y su consecuente configuración de violencias en la sociedad son transmitidas mayormente en la escuela. En ella, los/as individuos/as aprenden a ser varones y mujeres, además de los comportamientos adecuados por pertenecer a una $\mathrm{u}$ otra de estas categorías (Flores, 2005). Esto es transmitido a través de variados mecanismos, dentro de los cuales se encuentran medidas disciplinarias explícitas en torno al comportamiento adecuado de tal o cual género, pero también mediante procedimientos implícitos en el modo de funcionamiento y la malla curricular de cada establecimiento, lo cual es conocido como currículo oculto (Stromquist, 1998, en Flores, 2005). El resultado de esto son escuelas reproductoras y no preventoras de violencias de género, generando espacios no seguros tanto para población LGTBIQ+, como para hombres y mujeres que no calcen en el modelo estereotipado de género.

A partir de lo anterior, la presente investigación trató de aproximarse a las distintas expresiones de violencias de género en el espacio escolar y a las representaciones sociales de género que poseen los/as estudiantes secundarios/as de la Región Metropolitana. Para ello, se realizó una aproximación cualitativa inicial para, posteriormente, mediante el cruce de las observaciones cualitativas y el bagaje teórico previo, elaborar un cuestionario y obtener datos cuantitativos.

\section{APROXIMACIÓN TEÓRICA}

\subsection{ENFOQUE DE GÉNERO}

La teoría de género ha planteado la desigualdad dentro de las asignaciones sociales de género, en detrimento de la mujer y de lo que escape de la construcción masculina hegemónica. Ha develado una opresión invisibilizada históricamente y ha logrado posicionar la discusión acerca de la igualdad en planos que tradicionalmente eran soslayados por las teorías críticas. El llamado «enfoque de género» consiste en un cuestionamiento deconstructivista de nuestras identidades y de sus determinantes sociohistóricas. Se inscribe dentro de la comprensión de un modelo global de sexo-género (Rubin, 1996), y de cómo dicho modelo constituye una forma de dominio y control social a través de nuestros cuerpos y nuestra sexualidad. Como un ejemplo, el enfoque de género se puede preguntar qué hace que un cuerpo de determinada forma le sea asignada una personalidad y un rol específicos al interior de la sociedad: 
(...) Al existir hembras (o sea, mujeres) con características asumidas como masculinas y machos (varones) con características consideradas femeninas es evidente que la biología per se no garantiza tener las características de género. No es lo mismo el sexo biológico que la identidad asignada o adquirida; si en diferentes culturas cambia lo que se considera femenino o masculino, obviamente dicha asignación es una construcción social, una interpretación social de lo biológico; lo que hace femenina a una hembra y masculino a un macho no es pues la biología, el sexo, pues de ser así ni se plantearía el problema. (Lamas, 1986: 186)

Dicha determinación es completamente normalizada e incorporada de manera aproblemática, otorgándole a la estructuración de género un estatus biológico como forma de justificación (Lamas, 1989). Ahora bien, aquellas/os que han elaborado análisis y trabajado con un enfoque de género poseen muchas cosas en común; sin embargo, también existen muchas diferencias al interior de dicha perspectiva, históricamente levantada por el movimiento de mujeres feministas. Más allá de estas diferencias, lo importante es comprender que el enfoque de género nos invita a un cuestionamiento político en torno al dominio masculino que ha sido posibilitado por un sistema patriarcal, de carácter global y ancestral, que ejerce poder sobre los cuerpos y nos impone ciertas conductas como aceptables, lo cual es ante todo una herramienta para develar relaciones de poder, esto debido a que los atributos que asigna el género a las personas influyen sobre la organización de la sociedad en todas sus vertientes y sirven como base para la creación de jerarquías de poder (Arcos, Figueroa, Miranda y Ramos, 2007); de esta manera, «el género se constituye en un complejo mosaico de generación y reparto de poderes que se concretan en maneras de vivir y en oportunidades y restricciones diferenciales» (Arcos et al., 2007: 124).

\subsection{REPRESENTACIONES SOCIALES}

La teoría de las representaciones sociales corresponde a una tradición extraída de la psicología social, en particular del autor Serge Moscovici (1961), quien modifica la noción de representaciones colectivas de Durkheim, adaptándola para su uso en el análisis psicosociológico.

En Durkheim (1898), las representaciones colectivas son el pensamiento social incorporado en todas las personas. Sin embargo, Moscovici considera que, en las sociedades modernas, la división del trabajo social se complejiza y los escenarios de participación de las personas se diversifican, lo que genera que las representaciones varíen considerablemente dentro de un grupo social a otro. Es así como un mismo hecho puede producir distintas representaciones 
sociales, las cuales serán ajustadas según las expectativas y los deseos del sujeto/a que lo reciba (Jodelet, 1986).

Toda representación social es representación de algo y de alguien. No es lo real ni lo ideal, tampoco es subjetivo ni objetivo, sino que es siempre una relación del mundo con las cosas-objetos. Es decir, informaciones, imágenes, opiniones, actitudes que se relacionan con un objeto, a saber, un trabajo que realizar, un acontecimiento económico, un personaje social, etc. La representación varía según la posición que ocupan los/as sujetos/as en la sociedad, la economía y la cultura (Jodelet, 1986). Esto posibilita una multiplicidad de representaciones sociales, lo que se consolida con la sociedad de la información, en donde una gran parte de la población tiene acceso a una especificidad que le permite adquirir conocimientos que en el pasado se mantenían al alcance de solo un sector (Piña y Cuevas, 2004). Internet y los medios masivos de comunicación expanden las posibilidades de las representaciones sociales y se encargan de movilizar distintas combinaciones relacionales entre mundo-cosas en diferentes sectores de la sociedad.

Si bien Moscovici (1961) es quien posiciona el término de las representaciones sociales como una herramienta útil para el análisis de la interacción entre lo social y lo individual, el concepto recoge elementos y dialoga con distintas tradiciones de la teoría social que intenta explicar la internalización del mundo social en los/as individuos/as. De esta manera, una representación social es ante todo una construcción de sentido para la acción, o «una manera de interpretar y de pensar nuestra realidad cotidiana, una forma de conocimiento social» (Jodelet, 1986: 473), similar a la noción de «habitus» en Bourdieu, entendida como «esa especie de sentido práctico de lo que hay que hacer en una situación determinada» (Bourdieu, 1997: 40). Por ende, el conocimiento que aportan las representaciones sociales se enmarca en lo conocido como sentido común, el cual se aleja del conocimiento específico o científico (Jodelet, 1986). Este sentido común es una forma de conocimiento social que ha sido elaborado en colectivo y que permite organizar significativamente la realidad de la que los sujetos forman parte (Abric, 2001).

Es por esto que las representaciones sociales nos pueden ayudar a comprender cómo los estereotipos de género son transmitidos y cristalizados en sentidos comunes. Las representaciones sociales de género son transmitidas mediante la acción conjunta de múltiples instituciones basales de la sociedad familia, escuela, religión, entre otras-, las cuales determinan expectativas asociadas al comportamiento social, sexual e identitario de los cuerpos siguiendo la lógica binaria masculino-femenino. En otras palabras, los roles de género y todo lo que ello conlleva son transmitidos en forma de representación social, como un sedimento cultural que tributa al posicionamiento social del individuo: «La sexualidad, como cualquier otra acción, es una construcción social que responde a diversos elementos culturales, económicos e incluso 
políticos, que reflejan una concepción de mundo (...)» (Geertz, 1987, en Chávez, Vásquez y De la Rosa, 2007: 31).

Las representaciones sociales de género dibujan una barrera imaginaria de lo permitido, por ende, están sujetas a reproche y castigo en la medida que sobrepasen el límite de lo aceptado (Chávez, Vásquez, De la Rosa, 2007: 31). Es así como, por ejemplo, la sexualidad sin fines reproductivos ha estado siempre en el lado prohibido y punible de las prácticas sexuales. En esto colaboran no solo las instituciones valóricas como la Iglesia católica, que ha convertido el rechazo de la homosexualidad en «sentido común» para un sector de la población, sino que también la ciencia y en particular la psiquiatría y las instituciones de salud. El conocimiento erudito asimismo puede ser transformado en «sentido común», o bien puede ser nutrido de este mismo. Esto es un proceso que se compone de dos etapas: objetivización y anclaje (Jodelet, 1986). La objetivización, como el mecanismo mediante el cual lo social se transforma en representación; y el anclaje, mediante el cual la representación transforma lo social. Así, la Asociación Americana de Psiquiatría y la Organización Mundial de la Salud mantuvieron la homosexualidad como una patología hasta 1973 y 1990, respectivamente.

Por otra parte, las representaciones sociales de género tienen mucho que ver con los sentidos comunes que operan sobre el cuerpo de las mujeres. Nos estamos refiriendo a la representación de la mujer como ciudadana de segunda categoría, incapaz de cumplir cargos de alta responsabilidad, como única responsable de las labores reproductivas y domésticas; e incluso como origen de desgracias, perversiones y calamidades (Estramiana y Fernández, 2006: 71). Se pueden observar resabios de esto mediante el estudio y la observación del «chisme» como fenómeno social que recoge y cristaliza, a la vez, las representaciones sociales de género (Chávez, Vásquez y De la Rosa, 2007).

\subsection{VIOLENCIAS DE GÉNERO}

Queremos comprender los estereotipos de roles alimentados por las representaciones sociales, como formas de violencia simbólica. Es decir, que las expectativas sociales rígidas asociadas a los roles de género asignados al nacer devienen en violencia, en la medida que prohíben comportamientos, gustos, formas de vestir, etcétera; e imponen una manera binaria de constituir la identidad. Para comprender esto a cabalidad como una forma de violencia, es necesario complejizar nuestra mirada respecto a la violencia.

Lo primero es desprendernos de la noción superficial y reduccionista de la violencia, sobre todo en contextos escolares, en donde se entiende y reduce a la agresión física. Batsche y Knoff (1994) señalan que la violencia escolar ha sido definida tradicionalmente como actos de ataque, robo y/o vandalismo, debido a que es la parte observable del fenómeno y, por ende, fácil de documentar. Esta 
mirada es reduccionista, debido a que soslaya elementos fundamentales, tales como el dolor emocional y psicológico que puede resultar de un abuso de poder y también ignora los procesos sociales que hacen daño desde una perspectiva sistémica (Hall, 2000).

Es por esto que, para afinar nuestra mirada respecto a la violencia, debemos aproximarnos a ella de modo integral y expansivo. Esto implica reemplazar la idea de «fuerza» asociada a la violencia, por el concepto de «poder» y las relaciones simbólicas que rodean dicho fenómeno. La violencia, así, sería el ejercicio del poder para objetivar y «dañar» a otro/a. Una mirada integral y expansiva nos dice que el daño puede ocurrir en muchas dimensiones, más allá del daño físico o psíquico, el daño incluye las dimensiones de lo emocional, material, económico, social, identitario, moral, ético, etcétera (Batsche y Knoff, 1994). El poder se ejerce entre personas, pero también desde instituciones, comunidades, Estados-nación, etcétera. Siempre involucra una relación en donde existe un lado que ejerce el poder y otro que lo padece (sojuzgado). Las relaciones de poder están localizadas en el tiempo y el espacio, y no son sinónimo de represión, debido a que el poder construye y articula a la sociedad en su completitud, siempre a favor de quien lo ejerce (López, 2009).

Al tener esta compresión del fenómeno de la violencia, entendemos que puede ser ejercida de innumerables formas y en distintos planos. Al respecto, y focalizando nuestra mirada en el mundo juvenil del contexto escolar, Duarte y Aguilera (2009) reconocen tres planos de análisis en torno a la violencia entre jóvenes: i) estructural, ii) institucional y iii) situacional. Sobre la violencia estructural, se señala que son inherentes a un orden social legítimo, por ende, se valida en un uso de la fuerza legal para su existencia. Mientras que la violencia institucional se refiere a las formas en que distintos sectores de la sociedad ejercen poder sobre otros, para mantener sus privilegios, y, por último, la violencia situacional corresponde a situaciones cotidianas cuyos efectos aparecen en los imaginarios sociales y tienen efectos inmediatos (Duarte y Aguilera, 2009).

Para su uso dentro del género, Biglia y Jiménez (2015) proponen el uso de la noción «violencias de género», que representa «todas aquellas violencias que tienen su origen en una visión estereotipada de los géneros y en las relaciones de poder que esta conlleva o en las que se basan» (p. 27). Esta definición, según las autoras, permite incluir al género en sí mismo como configurador de violencias, en cuanto obliga a asumir roles, actitudes y comportamientos estereotipados que terminan causando discriminación social (Biglia y Jiménez, 2015). Sintéticamente, podemos decir que las violencias de género poseen cinco características: i) se encuentran amparadas en un sistema global de opresión de una cultura masculina hegemónica; ii) si bien pueden afectar a todos/as, se encuentran dirigidas principalmente hacia la población LGTBIQ+; iii) son normalizadas e invisibilizadas por el orden social; iv) son reforzadas y reproducidas por las instituciones fundamentales de la sociedad (por ejemplo, la 
escuela o la familia); y v) son violencias esencialmente simbólicas, por lo tanto, muchas veces no son problematizadas por la gran mayoría de quienes la sufren.

\subsection{VIOLENCIA ESCOLAR EN CHILE Y SU RELACIÓN CON LAS REPRESENTACIONES DE GÉNERO}

Las estadísticas levantadas por el IESSDEH durante 2011, en un estudio a través de Internet sobre el bullying y sus manifestaciones homofóbicas en escuelas de Chile, Guatemala, México y Perú, señalan que, en Chile, el 60,8\% de los/as encuestados/as reportó no haber recibido algún tipo de educación acerca de la homosexualidad o temas afines en su paso por la escuela. Además, el $68,4 \%$ de los/as estudiantes chilenos/as encuestados/as, con sexualidades disidentes a la norma, declararon enfrentar bullying. Al respecto, 42,1\% declaró ser víctima de bullying homofóbico frecuentemente y 36,8\% sufrir bullying homofóbico esporádico (IESSDEH y UPCH, 2011). Asimismo, cifras de la primera Encuesta de Clima Escolar aplicada por la Fundación Todo Mejora, dirigida a conocer experiencias de bullying y acoso escolar de jóvenes LGBTI+, señalan lo siguiente: $62,9 \%$ y $59,9 \%$ declaran haber sido acosados verbalmente debido a su orientación sexual o su expresión de género, respectivamente; mientras que $29,1 \%$ y $28,6 \%$ señalan haber sido agredidos/as físicamente en la escuela por las mismas razones, y $31,4 \%$ afirma haber sido acosado/a sexualmente (Fundación Todo Mejora, 2016).

Esta discriminación por orientación sexual y/o identidad de género no es propia de las escuelas, sino que es parte de las representaciones sociales de género que existen entre los/as jóvenes y que la escuela ayuda a reproducir. Por ejemplo, de acuerdo con datos del INJUV (2015), el 16,1\% de los y las jóvenes afirma que preferiría no tener como vecino a un transexual, a $10,9 \%$ no le gustaría que un homosexual o una lesbiana fuese su vecino/a, y a 10,4\% le desagrada la idea de ser vecino/a de una persona bisexual. También podemos encontrar que un $28,4 \%$ de las personas jóvenes afirma que «una pareja heterosexual, conformada por un hombre y una mujer, por lo general cría mejor a un niño que una pareja de personas del mismo sexo», y un $23,7 \%$ considera que «es preferible que los profesores de colegio sean heterosexuales en lugar de homosexuales» (INJUV, 2015).

Otra expresión común de las violencias de género ocurre en los contextos de pareja. Según cifras de la Policía de Investigaciones, 32\% de los y las jóvenes declara la presencia de señales de violencia en su relación de pareja, mientras que 9\% afirma haber sufrido violencia explícita (PDI, 2016). Estos datos coinciden con la información de la Octava Encuesta Nacional de Juventud, en donde el 13\% de las personas jóvenes señaló haber experimentado algún episodio de violencia psicológica en su relación de pareja, cifra que aumenta a $14 \%$ si contamos solo a las mujeres, y disminuye a $11,8 \%$ en el caso de los hombres. Respecto a víctimas de violencia física y sexual, 6,3\% fueron 
mujeres y $1 \%$ hombres. A esto se suma que $20,1 \%$ de los y las jóvenes indicó que su pareja controla sus salidas, horarios o apariencia, y 18,3\% reconoció haber realizado estas prácticas de control con su pareja (INJUV, 2015).

\section{MetOdOlOGíA Y TRABAJO DE CAMPO}

La selección de los casos que se trabajaron correspondió a criterios de representatividad según estratos identificados como relevantes en el contexto educativo de la Región Metropolitana: Ubicación (urbano-rural), Rendimiento (sobre la base de la PSU), Dependencia (municipal, subvencionado y particular) y Nivel Socioeconómico (sobre la base del SIMCE). En total, fueron seis establecimientos los participantes en el estudio, para lo cual contempló como universo la totalidad de matrículas de las escuelas involucradas en la investigación y se calculó un tamaño muestral de 598 casos, teniendo un margen de error del $2 \%$ y un nivel de confianza del $99 \%$.

La selección de los establecimientos operó en primera instancia sobre la base de los criterios definidos por las tipologías. Cumpliendo el establecimiento con los criterios de selección, se priorizaron aquellos con los cuales la Fundación ya hubiera trabajado, para así facilitar el acceso al campo y las relaciones institucionales. 

secundarios/as y violencias de género en la escuela

\begin{tabular}{|c|c|c|c|c|c|c|}
\hline & CASO 1 & CASO 2 & CASO 3 & CASO 4 & CASO 5 & CASO 6 \\
\hline Ubicación & Quilicura & $\begin{array}{l}\text { San José de } \\
\text { Maipo }\end{array}$ & $\begin{array}{l}\text { Santiago } \\
\text { Centro }\end{array}$ & $\begin{array}{l}\text { Santiago } \\
\text { Centro }\end{array}$ & La Florida & Peñalolén \\
\hline Tipología & $\begin{array}{l}\text { Urbano } \\
\text { Municipal } \\
\text { Mixto }\end{array}$ & $\begin{array}{l}\text { Rural } \\
\text { Municipal } \\
\text { Mixto }\end{array}$ & $\begin{array}{l}\text { Urbano } \\
\text { Municipal } \\
\text { Damas }\end{array}$ & $\begin{array}{l}\text { Urbano } \\
\text { Municipal } \\
\text { Varones }\end{array}$ & $\begin{array}{l}\text { Urbano } \\
\text { Particular } \\
\text { Subvencion } \\
\text { ado Mixto }\end{array}$ & $\begin{array}{l}\text { Urbano } \\
\text { Particular } \\
\text { Pagado } \\
\text { Mixto }\end{array}$ \\
\hline Matrícula $^{3}$ & $\begin{array}{l}280 \\
\text { estudiantes }\end{array}$ & $\begin{array}{l}424 \\
\text { estudiantes }\end{array}$ & $\begin{array}{l}2.884 \\
\text { estudiantes }\end{array}$ & $\begin{array}{l}942 \\
\text { estudiantes }\end{array}$ & $\begin{array}{l}1.100 \\
\text { estudiantes }\end{array}$ & $\begin{array}{l}482 \\
\text { estudiantes }\end{array}$ \\
\hline $\begin{array}{l}\text { Niveles de } \\
\text { enseñanza }\end{array}$ & $\begin{array}{l}\text { Educación } \\
\text { Prebásica y } \\
\text { Enseñanza } \\
\text { Básica }\end{array}$ & $\begin{array}{l}\text { Enseñanza } \\
\text { Media, } \\
\text { Técnico- } \\
\text { Profesional } \\
\text { Industrial y } \\
\text { Técnica, } \\
\text { Científica- } \\
\text { Humanista }\end{array}$ & $\begin{array}{l}\text { Enseñanza } \\
\text { Básica y } \\
\text { Media, } \\
\text { Científica- } \\
\text { Humanista }\end{array}$ & $\begin{array}{l}\text { Enseñanza } \\
\text { Media, } \\
\text { Científica- } \\
\text { Humanista }\end{array}$ & $\begin{array}{l}\text { Educación } \\
\text { Prebásica, } \\
\text { Enseñanza } \\
\text { Básica, } \\
\text { Media } \\
\text { Científica- } \\
\text { Humanista }\end{array}$ & $\begin{array}{l}\text { Educación } \\
\text { Prebásica, } \\
\text { Básica, } \\
\text { Media, } \\
\text { Científica- } \\
\text { Humanista }\end{array}$ \\
\hline $\begin{array}{l}\text { Caracterización } \\
\text { socioeconómica }{ }^{4}\end{array}$ & $\begin{array}{l}\text { GSE Medio } \\
\text { Bajo }\end{array}$ & $\begin{array}{l}\text { GSE Medio } \\
\text { Bajo }\end{array}$ & $\begin{array}{l}\text { GSE Medio } \\
\text { Alto }\end{array}$ & $\begin{array}{l}\text { GSE Medio } \\
\text { Bajo }\end{array}$ & GSE Medio & GSE Alto \\
\hline $\begin{array}{l}\text { Promedio PSU } \\
2^{2016^{5}}\end{array}$ & $\begin{array}{c}\text { NO } \\
\text { APLICA }\end{array}$ & 467 & 612 & 536 & 532 & 490 \\
\hline
\end{tabular}

La herramienta de recolección de datos consistió en un cuestionario autoaplicado durante la jornada escolar de cada establecimiento. Los y las estudiantes de cada establecimiento se eligieron de forma aleatoria, según el número de lista en cada curso de los niveles de séptimo básico a cuarto medio. La construcción del instrumento se basó tanto en categorías levantadas durante una etapa cualitativa previa, como también en otros cuestionarios aplicados por organismos e instituciones nacionales e internacionales, que trataran las temáticas de violencia escolar, convivencia escolar, igualdad de género, discriminación de población LGTBIQ+ $\mathrm{y}$ otros contenidos atingentes con nuestro objetivo de estudio.

\section{ANÁLISIS}

\subsection{CARACTERIZACIÓN DE LA MUESTRA}

Las edades de quienes participaron varían entre los 12 y los 19 años, encontrándose la media en 15,5 años. Las identidades de género de las/os

\footnotetext{
${ }^{3}$ El número de matrícula aproximado se basa en cifras de la plataforma digital MIME del Mineduc.

${ }^{4}$ La caracterización socioeconómica se basa en resultados SIMCE 2016.

${ }^{5}$ El promedio sobre la base de la información de la plataforma DEMRE.
} 
encuestados/as son en un 35,2\% masculinas, $62,4 \%$ femeninas, y un $1,8 \%$ se identifica con «otra» identidad de género. En su gran mayoría, quienes participaron son de nacionalidad chilena $(96,1 \%)$, sin embargo, se encuentran trece nacionalidades distintas, incluyendo la colombiana, haitiana, peruana y ecuatoriana, entre otras. Residen en veintiséis comunas distintas, encontrándose la mayor parte de ellos en la comuna de La Florida (18\%), seguido por Santiago (7\%), Maipú (6,7\%), Quilicura (5,9\%), San José de Maipo (5,7\%) y Pudahuel $(5,4 \%)$. En otros datos relevantes, el $1 \%$ de la muestra declaró ser padre o madre, y el 10,9\% participa de algún tipo de organización.

La orientación sexual de las/os encuestadas/os fue obtenida a partir de las preguntas «identidad de género» y «Normalmente sientes atracción física y amorosa por...». A partir de ello, pudimos obtener que las muestra se encuentra representada en un $70,7 \%$ por estudiantes heterosexuales; $19,7 \%$ por mujeres bisexuales; $2,2 \%$ por hombres bisexuales; $2 \%$ de hombres homosexuales; y un $1,8 \%$ de mujeres homosexuales. Un $3,6 \%$ de la muestra se encuentra indefinido/a en su preferencia sexual (optan por la alternativa «no lo tengo claro»).

Si bien la muestra contiene un número mayor de representación femenina, las mujeres muestran una tendencia marcada a pertenecer a orientaciones sexuales no heterosexuales, más que los hombres. El 33,5\% de las mujeres versus el $11,6 \%$ de los varones poseen una orientación sexual no heterosexual, como se aprecia en la siguiente tabla. Dicho de otra manera, tres de cada diez mujeres no son heterosexuales, versus uno de cada diez hombres.

\begin{tabular}{lrrr}
\hline & Heterosexual & \multicolumn{1}{c}{ No } & Indefinido/a \\
heterosexual & \\
Masculina & $83,9 \%$ & $11,6 \%$ & $4,5 \%$ \\
Femenina & $62,8 \%$ & $33,5 \%$ & $3,7 \%$ \\
Otra &, $0 \%$ &, $0 \%$ & $100,0 \%$ \\
\hline
\end{tabular}

\subsection{REPRESENTACIONES SOCIALES DE GÉNERO}

Las RSG se abordaron durante el estudio en tres dimensiones: i) valoraciones asociadas a las desigualdades estructurales que sustentan las relaciones desiguales de género; ii) la rigidez en torno a los estereotipos de género y iii) tolerancia a la diversidad sexual y expresiones de género. Para cada dimensión se elaboraron una serie de afirmaciones que debían ser respondidas con una escala de Likert. Luego, con las preguntas se generó un índice que agrupaba las respuestas en tres categorías: RSG inclusivas, RSG neutras y RSG conservadoras. 


\section{Desigualdades estructurales}

Esta dimensión contempla posicionamientos respecto a los obstáculos sistémicos que enfrentan las mujeres y que sustentan desigualdades de género respecto a los hombres, tales como la prohibición al aborto, la violencia física, verbal y psicológica, las desigualdades respecto a salario y valoración de sus capacidades para desempeñar cargos.

La variable con mayores representaciones conservadoras en esta dimensión fue la referida al aborto. El 32,7\% no se muestra favorable a la legalización del aborto en cualquier circunstancia. Sin embargo, el $67,3 \%$ de los y las estudiantes piensa que debiese legalizarse en cualquier causal. El 20,3\% de la muestra señala que la píldora del día después no debiese ser entregada a cualquier mujer en edad reproductiva que la solicite. Luego, el 13,9\% cree que en ciertas circunstancias la violencia ejercida en contra de la mujer se encuentra justificada. El 10,2\% piensa que las mujeres deben evitar ropa que provoque a los hombres; el 8,5\% que es normal que las mujeres ganen menos que los hombres, y un 5,8\% que las mujeres no tienen las mismas capacidades para desempeñar cargos de alta responsabilidad. El promedio de RSG conservadoras en esta dimensión es de $15,2 \%$.

\section{Rigidez de estereotipos de género}

Esta dimensión se compone de actitudes y valoraciones que sitúan la identidad femenina cristalizada en labores tradicionalmente asociadas a su género, tales como la crianza y las labores domésticas y, por otro lado, que sitúan al hombre en un rol proveedor, dominante y violento.

En esta dimensión, la variable con mayores representaciones no-inclusivas refiere a la maternidad, en donde el $27,4 \%$ de la muestra señala que, en general, las mujeres que tienen hijos/as se sienten más realizadas que quienes no los/as tienen. Reforzando la vinculación de la construcción de lo femenino, desde las representaciones asociadas al cuidado y la maternidad. Por otra parte, el 23,9\% de la muestra señala que para los hombres es muy difícil el controlar sus impulsos sexuales, reforzando por el lado de la masculinidad, la idea de impulsos sexuales incontrolables.

El 16,1\% piensa que los hombres son violentos por naturaleza; el 12,9\% de la muestra señala que le resulta desagradable ver a un hombre comportándose «como una mujer»; el 12,9\% también dice que la mujer es quien debe preocuparse de usar un método anticonceptivo durante el sexo; el 10,7\% considera que es tarea del hombre mantener económicamente a la familia; al $10,6 \%$ le resulta molesto ver a una mujer comportándose «como un hombre»; el $7,9 \%$ piensa que el cuidar a los/as hijos/as es tarea de la mujer, y solo el $4,8 \%$ cree que las mujeres deben tener relaciones sexuales cuando su pareja se los pide. El promedio de RSG conservadoras en esta dimensión es de 14,1\%. 


\section{Tolerancia a la diversidad sexual}

Esta última dimensión se compone de posicionamientos respecto a valoraciones-actitudes que comprenden la sexualidad como heteronormada, y la identidad-expresión de género como binaria y determinada por la biología.

El 17,3\% de la muestra señaló que prefiere que los profesores sean heterosexuales. Luego, el 16,6\% considera que un verdadero hombre solo tiene relaciones sexuales con mujeres, y el $15,4 \%$, que una verdadera mujer solo tiene relaciones con hombres. El 13,2\% no cree en el matrimonio entre personas del mismo sexo y el $11,2 \%$ piensa que la homosexualidad no es normal ni natural. El 10,8\% de la muestra se siente incómodo estando al lado de un hombre homosexual, y un 8,8\%, al lado de una mujer homosexual. El $8 \%$ no cree que se les debiese dejar adoptar a las parejas homosexuales, y un 5,2\% que se les debe prohibir trabajar con niños/as. Por último, el $4,1 \%$ de la muestra señala que jamás tendría un amigo o amiga homosexual. El promedio de RSG conservadoras en esta dimensión es de $11,1 \%$.

\subsection{VIOLENCIA INSTITUCIONAL}

Con el análisis de la violencia institucional nos referimos a los procedimientos y mecanismos institucionales, pero también a las prácticas, actitudes y/o comentarios de parte de los/as funcionarios/as del establecimiento frente a: i) el respeto hacia la diversidad sexual e identitaria; ii) visibilización de la diversidad sexual y educación sexual; iii) reproducción de estereotipos de género y iv) presencia de violencia sexual. En esta parte del análisis se contemplan las variables del cuestionario que refieren ya sea a la institución, como a autoridades o funcionarios del establecimiento.

\section{Respeto a la diversidad sexual}

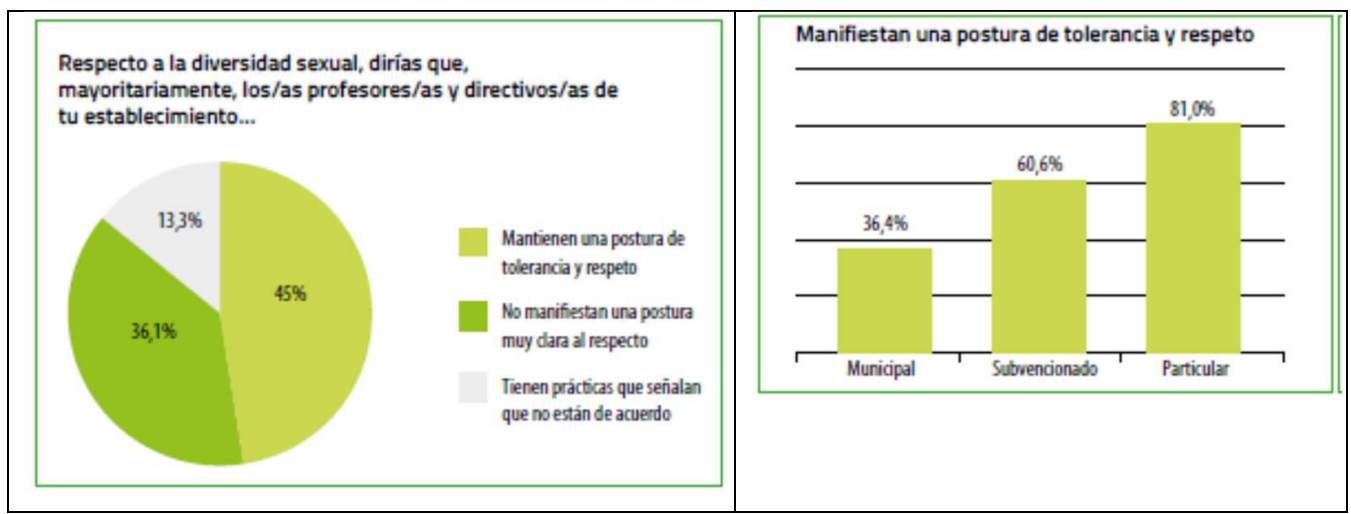


Según la percepción de los/as estudiantes, casi la mitad de los/as profesores/as y directivos/as de sus establecimientos no tienen una postura clara respecto a la diversidad sexual, y/o manifiestan una postura contraria a ella, versus un $45 \%$ que mantiene una postura de tolerancia y respeto.

Podemos observar que esto se agrava en el caso de los establecimientos municipales y se matiza en el caso de los particulares. Solo el $36,4 \%$ de los docentes y directivos de los establecimientos municipales manifiestan una postura de tolerancia y respeto, versus el $81 \%$ en el establecimiento particular.

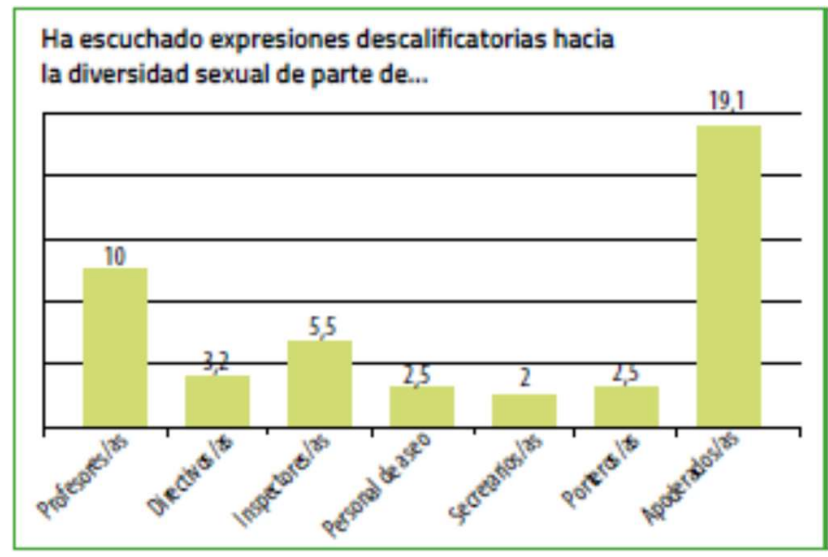

Profundizando la mirada, el $10 \%$ de la muestra ha escuchado expresiones descalificatorias hacia la diversidad sexual de parte de profesores/as, el 5,5\% de parte de inspectores y el $3,2 \%$ de directivos/a. Sin embargo, los apoderados/as aparecen como el actor que más expresa comentarios descalificatorios, con un

$19,1 \%$ de la muestra que declara que ha escuchado este tipo de expresiones de parte de ellos/as.

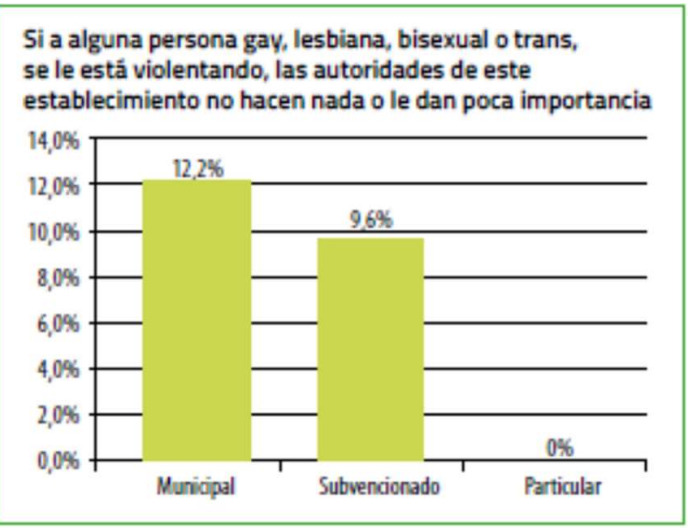

La percepción de la importancia que le dan las autoridades de los establecimientos respecto al bullying LGTBIQ+ o a la violencia que reciben estos grupos varía significativamente al interior de las dependencias de los establecimientos, siendo más alta la percepción de indolencia respecto a estos temas en los establecimientos públicos. 


\section{Visibilización de las disidencias sexuales y educación sexual}

En los establecimientos municipales, el $58,4 \%$ de quienes participaron declararon recordar actividades en donde había sido abordada la temática de la diversidad sexual, porcentaje mayor a los establecimientos particular $(39,5 \%)$ y subvencionado $(32,7 \%)$, lo cual constituye un revés en la tónica de la brecha observada hasta ahora. Esto se puede explicar por programas externos al establecimiento que ofrecen capacitación e información en estas materias, lo cual fue observado durante el proceso etnográfico de la etapa cualitativa, o también puede deberse a las múltiples actividades organizadas por colectivos de estudiantes pertenecientes a los establecimientos municipales para tratar la temática. Sin embargo, no representa necesariamente una política institucional de visibilizar la diversidad sexual al interior de sus contenidos.

Al preguntar respecto a la educación sexual, pese a que esta ha recibido un impulso en los últimos años a lo largo del país, con las reformas educacionales, el porcentaje de estudiantes que declara no haber recibido educación sexual sigue siendo alarmante, con un $27 \%$ de la muestra general, porcentaje que aumenta a $29,6 \%$ en el caso de los establecimientos públicos.

Y respecto a quienes han recibido educación sexual, existe un porcentaje importante de estudiantes $(25,9 \%)$ que declaran que la educación sexual no les ha servido en sus vidas. Solo el $41,9 \%$ de quienes participaron dijeron haber recibido educación sexual y estar conforme con ella.

\section{Reproducción de estereotipos de género por parte de personal del establecimiento}

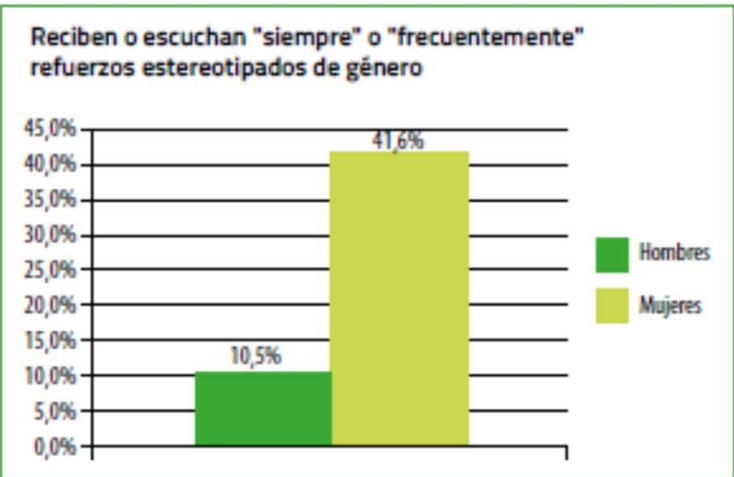

En esta dimensión se ve una alarmante brecha entre hombres y mujeres. Podemos observar que las mujeres reciben más refuerzos de género estereotipados que los hombres, en una razón de 4 a 1. Este tipo de refuerzos son comentarios que asumen una forma rígida de femineidad o masculinidad. En el caso de las

mujeres, se traduce en expresiones como «una señorita no hace esas cosas» o «compórtese como una dama». En el caso de los hombres, «los hombres no lloran», o bien, «compórtate como hombre». 


\section{Violencia sexual}

Otra brecha de género importante aparece al interior de quienes han recibido violencia sexual dentro del establecimiento. La violencia sexual involucra: tocaciones no consentidas, abuso sexual, insinuaciones sexuales no consentidas, divulgación de fotografías íntimas y miradas lascivas. El 75,3\% de la muestra no ha recibido ni una de estas violencias al interior de sus establecimientos. Sin embargo, el 24,7\% declara haber recibido al menos una de estas violencias de tipo sexual. Este tipo de violencia no se encuentra igualmente distribuida según identidad de género, siendo las identidades no binarias las más afectadas $(36,4 \%)$, seguidas de las mujeres $(27,2 \%)$.

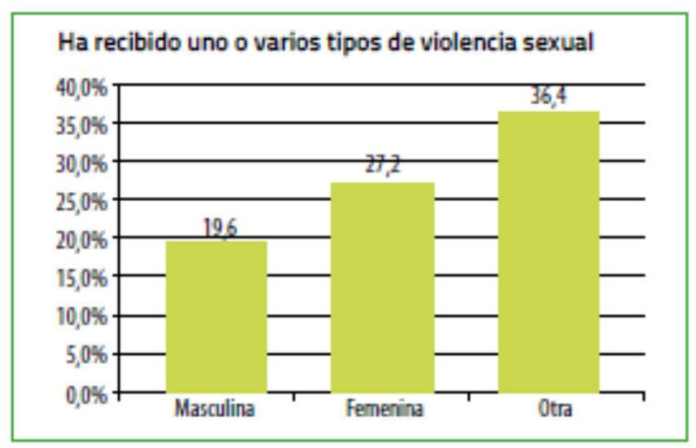

\section{Apertura Reflexiva}

Podemos notar que la mayor cantidad de representaciones conservadoras se encuentran al interior de la dimensión de desigualdades estructurales. Dentro de esta dimensión, el aborto y la píldora del día después pueden ayudar a explicar dicha concentración. La discusión sobre el aborto se encuentra muy presente en el país después de la aprobación de la ley que lo legaliza en tres causales, lo que ha generado una visibilización del debate a nivel país, y una polarización de las posturas frente al tema. Al respecto, podemos señalar que, si bien la brecha entre hombres y mujeres no es tan distanciada como en otras variables, sí se nota una mayor recepción al aborto en las mujeres que en los hombres, y una adhesión al aborto de un $100 \%$ en el caso de las identidades no binarias.

La distribución general de RSG inclusivas, intermedias y conservadoras se situó en $78,3 \%, 18,9 \%$ y 2,8\%, respectivamente. La gran mayoría de las/os estudiantes tienen apertura respecto a la diversidad sexual, y también cuestionan los roles estereotipados asociados a la masculinidad y a la femineidad. Sin embargo, al igual que en el resto de las variables, no se encuentran igualmente distribuidas entre los establecimientos. 


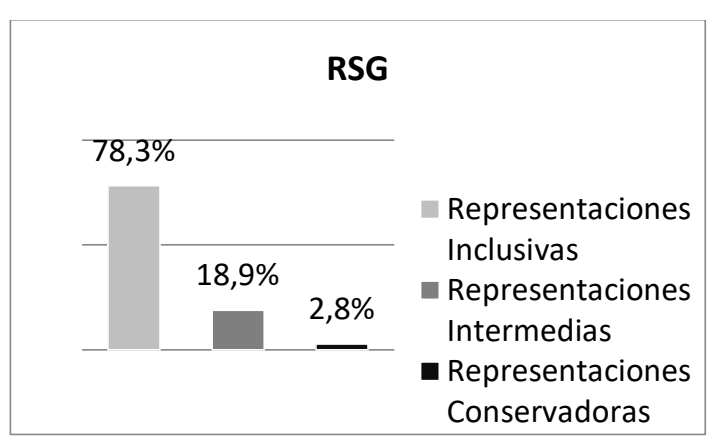

\begin{tabular}{lccc}
\hline Representaciones socioculturales de género v/s dependencia & \\
& $\begin{array}{c}\text { Representaciones } \\
\text { inclusivas }\end{array}$ & $\begin{array}{c}\text { Representaciones } \\
\text { intermedias }\end{array}$ & $\begin{array}{c}\text { Representaciones } \\
\text { conservadoras }\end{array}$ \\
Municipal & $82,1 \%$ & $15,9 \%$ & $2,1 \%$ \\
Subvencionado & $59,8 \%$ & $35,9 \%$ & $4,3 \%$ \\
Particular & $97,1 \%$ & $2,9 \%$ &, $0 \%$ \\
Total & $78,8 \%$ & $18,8 \%$ & $2,4 \%$ \\
\hline
\end{tabular}

Al ver la distribución por dependencia, nos encontramos con que en el establecimiento subvencionado se concentra mayor porcentaje de estudiantes con representaciones intermedias y conservadoras. Esto puede deberse a la educación valórica del establecimiento, asociada a una mirada religiosa. Además de las otras particularidades del caso que lo posicionan desde un paradigma conservador en cuanto a su orientación valórica.

Sin embargo, al ver la distribución según la ubicación del establecimiento, vemos que existe otra variable que logra explicar de mejor manera la distribución de estudiantes y sus representaciones de género, pues el establecimiento ubicado en una zona rural posee un $48,5 \%$ de estudiantes con representaciones conservadoras e intolerantes.

\begin{tabular}{lccc}
\hline & \multicolumn{3}{c}{ Representaciones socioculturales de género v/s ubicación } \\
& $\begin{array}{c}\text { Representaciones } \\
\text { inclusivas }\end{array}$ & $\begin{array}{c}\text { Representaciones } \\
\text { intermedias }\end{array}$ & $\begin{array}{c}\text { Representaciones } \\
\text { conservadoras }\end{array}$ \\
Urbano & $80,9 \%$ & $17,1 \%$ & $2,1 \%$ \\
Rural & $51,5 \%$ & $42,4 \%$ & $6,1 \%$ \\
Total & $78,8 \%$ & $18,8 \%$ & $2,4 \%$ \\
\hline
\end{tabular}

La variable orientación sexual también logra explicar en alguna medida la distribución de RSG, existiendo solo un $0,8 \%$ de encuestados/as no heterosexuales con RSG conservadoras. 


\begin{tabular}{lccc}
\hline & \multicolumn{3}{c}{ Representaciones socioculturales de género } \\
& $\begin{array}{c}\text { Representaciones } \\
\text { inclusivas }\end{array}$ & $\begin{array}{c}\text { Representaciones } \\
\text { intermedias }\end{array}$ & $\begin{array}{c}\text { Representaciones } \\
\text { conservadoras }\end{array}$ \\
Heterosexual & $72,0 \%$ & $24,1 \%$ & $4,0 \%$ \\
No heterosexual & $93,7 \%$ & $5,6 \%$ &, $8 \%$ \\
Indefinido/a & $80,0 \%$ & $20,0 \%$ &, $0 \%$ \\
Total & $78,1 \%$ & $19,0 \%$ & $2,9 \%$ \\
\hline
\end{tabular}

Es importante destacar los altos porcentajes de violencias sexuales en estudiantes, así como la presencia de refuerzos de estereotipo de género significativamente más recurrentes en mujeres que en hombres. Pero también, las violencias tienen una clara diferenciación con respecto a los establecimientos educacionales, en donde las mayores concentraciones de medios y altos índices de violencia se sitúan en establecimientos de dependencia municipal y particular subvencionado. Si bien los establecimientos municipales han realizado mayores esfuerzos por la visibilización de las disidencias sexuales, se debe destacar que estos muestran, de acuerdo con la tabla anterior, las mayores relaciones de violencia.

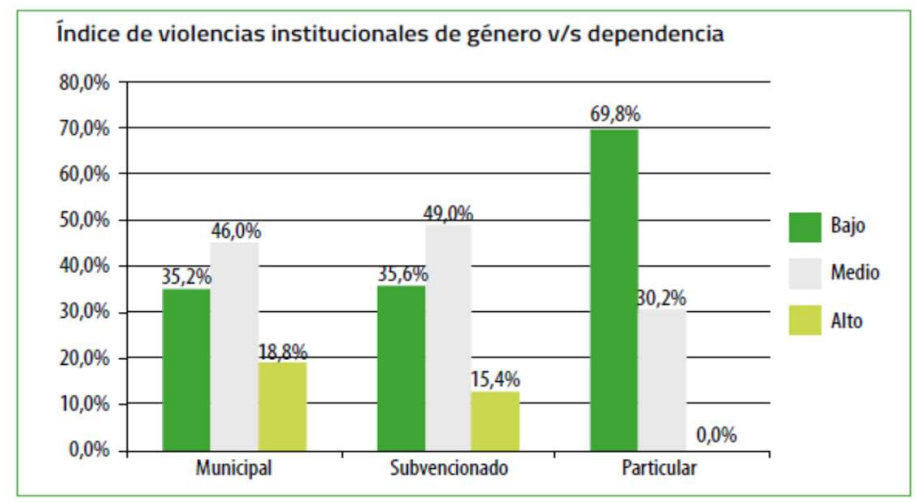

\section{CONCLUSIÓN}

Las violencias de género son una categoría analítica que nos permite introducirnos en indicadores sutiles, pero profundos, que afectan tanto la convivencia escolar como la calidad de vida de quienes habitan la escuela, lo que finalmente determina la calidad de la educación de los establecimientos y, también, la posibilidad de construir una escuela participativa, inclusiva y democrática.

La muestra trabajada a lo largo de la investigación buscó representar distintas realidades de establecimientos educacionales del país, por ende, existen escenarios muy diversos al interior de los establecimientos con los cuales trabajamos. Sin embargo, en todos ellos existen manifestaciones 
importantes de violencias de género. Lo que cambia es la importancia con la cual se trata el tema, los recursos, procedimientos y mecanismos con los cuales cuenta el establecimiento para solucionar situaciones particulares, el capital cultural de los/as estudiantes y el entorno social de la comunidad escolar que inserta a sus miembros/as en contextos de desposesión material, o estructuras cotidianas de violencia sistémica.

Respecto a las diferencias, esta investigación ha podido visibilizar las brechas existentes en distintos tipos de variables entre establecimientos municipales versus el establecimiento subvencionado y el particular. Si bien todos poseen grados de violencias de género al interior de sus comunidades, podemos señalar que es en los establecimientos de dependencia municipal en donde se dieron las principales diferencias respecto al resto de las tipologías. El nivel de conflictividad, sobre todo en el caso de los conflictos entre estudiantes y profesores, así como la efectividad de los mecanismos para resolver los conflictos, se ve de forma más crítica al interior de los establecimientos municipales, en donde la conflictividad es mayor y la percepción de la efectividad de los mecanismos es menor.

En cuanto a los mecanismos de procedimiento, en caso de burlas y/o discriminaciones, los establecimientos municipales toman menos medidas $(23,3 \%)$ que el establecimiento subvencionado $(33,7 \%)$ y el particular $(39,5 \%)$. $\mathrm{Y}$ en el caso del establecimiento privado, estas medidas obtienen mayores grados de satisfacción que en los municipales y subvencionados. Esto se replica en los casos de violencia hacia población LGTBIQ+, en donde el porcentaje de estudiantes que declara que el establecimiento «no hace nada» en estos casos aumenta en la dependencia municipal. Por otra parte, los profesores/as de los establecimientos municipales tienen menos gestos de mostrar apertura y tolerancia a la diversidad sexual, que en el caso de los establecimientos subvencionado y particular.

La educación sexual también posee una brecha importante entre tipos de establecimiento, siendo más común en los establecimientos particular y subvencionado de la muestra. El $29,6 \%$ de los y las estudiantes de establecimientos municipales declara no haber recibido educación sexual, frente al $20,2 \%$ en el establecimiento subvencionado, y el $14 \%$ del particular. Las brechas en la calidad de la educación municipal, que ha sido visibilizada y puesta en el foco de la atención pública por el movimiento estudiantil, no solo poseen distancia respecto a la educación privada en los resultados de pruebas estandarizadas, o de acceso a la educación superior, sino que se expresa en indicadores de calidad sustanciales para la vida de las y los estudiantes, que les dejan expuestos a otro tipo de vulnerabilidades. Esta investigación ha podido visibilizar algunas de las principales aristas que diferencia a los tipos de colegio desde la mirada de la educación de género.

$\mathrm{Al}$ entender las violencias de género como una realidad cultural presente en todas las esferas de la sociedad, nos encontramos frente a un problema 
transversal y cuya pregunta fundamental no es ¿se encuentra presente?, sino ¿qué características adquieren? Tal como hemos visto en nuestro recorrido por la teoría que sustenta esta investigación, la escuela juega un papel fundamental en la reproducción de las representaciones sociales de género, por ende, puede llegar a jugar también un rol importante en su desnaturalización. Es en las escuelas donde la socialización de género obtiene gran parte de su proceso, y en donde ocurren discriminaciones y violencias que pueden determinar la vida de una persona.

Las violencias de género deben abordarse en las escuelas como una problemática urgente que debe ser enfrentada con perseverancia, humildad y apertura, debido a que significa una perspectiva que despertará la incomodidad de un gran sector del establecimiento. Para esto, es fundamental no solo la capacitación del personal docente y directivo, sino también la elaboración de un plan estratégico que se adapte a la realidad y necesidad de cada comunidad. Esta investigación permite contar con instrumentos concretos para caracterizar las violencias de género, y así trazar una carta de navegación que se adapte a los puntos sensibles de la comunidad.

RECIBIDO: 15 DE MAYO DE 2019

ACEPTADO: 18 DE JUNIO DE 2019 


\section{BibLIOGRAFÍA}

ABRIC, J. C. (2001). «Las representaciones sociales: aspectos teóricos». En Prácticas sociales y representaciones (pp. 7-32). México, D.F.: Ediciones Coyoacán.

Arcos, E., Figueroa, V., Miranda, C. y Ramos, C. (2007). «Estado del Arte y Fundamentos para la construcción de indicadores de género en la educación». Estudios Pedagógicos, 121-130.

BATSCHE, G. M. y KNOFF, H. M. (1994). «Bullies and their victims: Understanding a pervasive problem in the schools». Schoool Psychology Review (23): 165-175.

Biglia, B. y JimÉNEZ, E. (2015). Jóvenes, género y violencias: Hagamos nuestra la prevención. Tarragona: Universitat Rovira i Virgili/Publicaciones URV.

Bourdieu, P. (1997). «El nuevo capital». En Razones Prácticas. Sobre la teoría de la acción. Madrid: Anagrama.

Chávez, M. E., VÁsquez, V. y De la Rosa, A. (2007). «El chisme y las representaciones sociales de género y sexualidad en estudiantes adolescentes». Perfiles Educativos, XXIX, 115: 21-48.

DuARte, C. y Aguilera, O. (2009). «Juventud y violencia». Revista Observatorio de Juventud, 6, 23.

DURKHEIM, É. (1898). «Représentations individuelles et représentations collectives». En Sociologie et Philosophie. París: PUF, 1967.

ESTRAmiAnA, J. L. y FERnÁNDEZ, B. (2006). «Representaciones sociales de la mujer». Athenea Digital, 9: 65-77.

FLORES, J. I. (2003). «La perspectiva cultural de la organización escolar-marco institucional y comportamiento individual». Educar, 109-119.

Flores, R. (2005). «Violencia de género en la escuela: sus efectos en la identidad, en la autoestima y en el proyecto de vida». Revista Iberoamericana de Educación, 38: 67-86.

Fundación Todo Mejora Chile. (2016). «Encuesta Nacional de Clima Escolar en Chile 2016. Experiencias de niños, niñas y adolescentes lesbianas, gays, bisexuales, y trans en Establecimientos Educacionales». Disponible en: https://todomejora.org/wpcontent/uploads/2016/08/Encuesta-de-Clima-Escolar-2016-FundacionTODOMEJORA.pdf

HaLl, S. (2000). «What is School Violence? An integrated Definition». The Annals of the American Academy of Political and Social Science, 567: 16-29. 
IESSDEH y UPCH. (2011). Instituto de Estudios en Salud, Sexualidad y Desarrollo Humano/Universidad Peruana Cayetano Heredia, en colaboración con la Organización Panamericana de la Salud. Lima: 2011. Disponible en:

http://www.iessdeh.org/usuario/ftp/Informe\%20Final\%20Bullying.pdf

INJUV. (2015). «Octava Encuesta Nacional de la Juventud 2015». Ministerio de Desarrollo Social. Disponible en: http://www.injuv.gob.cl/portal/wpcontent/uploads/2017/03/libro-octava-encuesta-nacional-dejuventud.pdf

JODELET, D. (1986). «La representación social: fenómenos, concepto y teoría». En S. Moscovici (Comp.), Psicología social II. Pensamiento y vida social (pp. 469-493). Barcelona: Paidós.

LAMAS, M. (1986). «La antropología feminista y la categoría "género"». Nueva Antropología, VIII: 173-198.

LóPEz, F. L. (2009). Poder y dominio (1 ${ }^{\mathrm{a}}$ edición). Buenos Aires: Madreselva.

MoscovicI, S. (1961). El psicoanálisis, su imagen y su público. Buenos Aires: Huemul.

PDI. (2016). Encuesta «No más violencia en el pololeo». Policía de Investigaciones de Chile. Disponible en:

https://www.policia.cl/jenafam/sitio_jenafam/jenafam/descargas/archivo s/violenciapololeo/

Resultados\%20Encuesta\%202016,\%20No\%20+\%20Violencia $\% 20$ en $\%$ 20el\%20Pololeo.pdf

PiÑA, J. M. y Cuevas, Y. (2004). «La teoría de las representaciones sociales: Su uso en la investigación educativa en México». Perfiles Educativos, 26(105-106): 102-124. Disponible en: http://www.scielo.org.mx/scielo.php?script=sci_arttext\&pid=S018526982004000100005\&lng=es\&tlng=es.

RUBIN, G. (1996). «El tráfico de mujeres: notas sobre la "economía política" del sexo». En M. LAMAS (Comp.), El género: la construcción cultural de la diferencia sexual. México, D.F.: UNAM. 